\title{
NOVEL WATERMARKING METHODS BASED ON FREQUENCY DOMAIN AND SINGULAR VALUE DECOMPOSITION
}

\begin{abstract}
Nowadays, the copyright laws protecting the digital content are getting quite common. One of the important things is to protect the original or compressed images against illegal use. Frequency domain watermarking methods are used in most cases in today's image- and videowatermarking algorithms. Especially Discrete Wavelet Transform (DWT) and Discrete Cosine Transform (DCT) are used in watermarking algorithms. This paper deals with development of two novel digital image watermarking methods that use DWT with Singular Value Decomposition (SVD) and DCT with SVD. The paper includes step by step process of watermark embedding and extraction and testing of the watermark robustness. The robustness was tested with the Checkmark, one of the watermark benchmarking tools. Experimental results show that both schemes are robust against a lot of attacks and have very high data hiding capacity.
\end{abstract}

Keywords: Singular value decomposition, frequency domain, robustness, watermark.

\section{Introduction}

Nowadays, most of digital images are stored in the digital form for future utilization. The digital data protection against illegal use becomes still more important. In the field of digital images, the protection against copying or modifications is most important. One way how to protect digital images is to use digital watermarking techniques. There exist two general ideas: the visible watermark and the invisible watermark. Visible watermarks are mainly known for example as logos of television stations. The invisible watermarks are used especially for author rights authentication to the digital content (image, video). Generally speaking, invisible watermarks can be inserted in the spatial domain or in the transform domain [1]. There exist lots of methods which use the spatial domain for watermark insertion [2] and [3]. The most famous and firstly published method uses the least significant bits of the image data for watermark insertion. However, most of these methods are not robust enough.

This paper focuses on the invisible watermarks and techniques that use the transform domain of digital images for watermark insertion and are useful, for example, to secure the images in biomedical systems [4].

Discrete Cosine Transform (DCT), Discrete Fourier Transform (DFT), Discrete Wavelet Transform (DWT) and Walsh-Hadamard Transformation (WHT) are used to transform from spatial to frequency domain. These transforms are mostly used in many frequency domain watermarking methods [5], [6], [7] and [8].
This paper deals with watermarking methods that use DWT, DCT and SVD together. We developed two novel techniques that are robust to various types of attacks. The first technique is based on 2D-DCT and SVD transforms, the second one is based on 2DDWT and SVD. Experimental results described in last sections show that both of these methods are suitable for image data protection against illegal use.

\section{Common techniques used in watermarking systems}

In most cases the frequency domain representation of the digital image is acquired using two dimensional DCT (2D-DCT) or two dimensional DWT (2D-DWT). The 2D-DCT is usually applicable on the square image data (matrix) of dimension $8 \times 8$ pixels. Similarly to other transforms, the 2D-DCT attempts to decorrelate the image data. Low frequencies are concentrated in the top left corner of the transformed matrix, high frequencies are concentrated in the bottom right corner. This transform is used in the well-known JPEG and MPEG compressions schemes. The second frequently used transform in image and video compressions schemes is the 2D-DWT. The 2D-DWT decomposes the original image into four bands, the watermark can be embedded in all frequencies [7].

SVD as a general linear algebra technique is used in a variety of applications, for example in watermarking. Modifying the singular value decomposition of the image is one of the most prevalent techniques in transform domain watermarking. SVD is described by the equation

\footnotetext{
* Petr Cika, Martin Zukal

Department of Telecommunications, Faculty of Electrical Engineering and Communication, Brno University of Technology, Czech Republic E-mail: cika@feec.vutbr.cz
} 


$$
A=U S V^{T}
$$

where the diagonal entries of $S$ matrix are the singular values, the $U$ matrix contains the left singular vectors and the $V$ matrix contains the right singular vectors. Each of the new matrices has the same dimension as the original matrix $A$. The important fact is that each singular value $\mathrm{s}$ from the equation

$$
S=\left(\begin{array}{ccc}
s_{1} & \cdots & 0 \\
\vdots & \ddots & \vdots \\
0 & \cdots & s_{i}
\end{array}\right)
$$

specifies the luminance value of image layers while the corresponding pair of singular vectors specifies the geometry of the image layer [9].

\section{Quality parameters}

The Peak Signal to Noise Ratio (PSNR) was used for testing the quality of the image with embedded watermark. The final PSNR value is expressed as

$$
P S N R=10 \log _{10}\left(\frac{255^{2}}{M S E}\right)
$$

In case of gray-scale images. MSE is Mean Square Error defined by the equation

$$
M S E=\frac{1}{M N} \sum_{m=0}^{M-1 N-1} \sum_{n=0}\left[x(m, n)-x^{\prime}(m, n)\right]^{2}
$$

where $M, N$ define the image size, $x$ is the pixel value of the original image, and $x^{\prime}$ is the pixel value of the watermarked image.

The Normalized Cross Correlation (NCC) function was used for quality evaluation of the extracted watermark. NCC is defined by the equation

$$
N C C=\frac{\sum_{i=0}^{I-1} \sum_{j=0}^{J-1} W_{i j} W_{i j}^{\prime}}{\sum_{i=0}^{I-1} \sum_{j=0}^{J-1}\left[W_{i j}\right]^{2}}
$$

where $I, J$ define the size of the embedded watermark and $W, W^{\prime}$ define the original and extracted watermark bits.

\section{Proposed watermarking methods}

New watermarking schemes based on a method described in [5] will be presented in the following paragraphs. The first proposed method uses 2D-DWT and SVD, the second one uses 2DDCT and SVD. Both methods divide the original image I into four sub-images before embedding.

The following steps describe the embedding process of the proposed watermarking methods (Fig. 1):

- The first step is to divide the gray-scale image $I$ into 4 subimages $I_{1}, \ldots, I_{4}$,
- 2D-DWT or 2D-DCT is applied on each sub-image $I_{1}, \ldots, I_{4}$ (only the first decomposition is used in case of 2D-DWT),

- in the next step the SVD is used:

- 2D-DWT: SVD is used to CA sub-band.

- 2D-DCT: All coefficients are zig-zag scanned at first, the SVD is used only for the first quarter of DCT coefficients.

The SVD is computed by the equation

$$
A_{k}=U_{k} S_{k} V_{k,}^{T}, \quad k=1,2,3,4
$$

where $k$ specifies the sub-image.

- The 2D-DWT/2D-DCT is applied on the whole watermark image $W$. Dimensions of the watermark image must be equal to quarter of the size of the original image $I$.

- Next the SVD is applied. The CA sub-band of the watermark $W$ is processed in case of 2D-DWT. The first quarter of zig-zag scanned DCT coefficients is used in case of 2D-DCT:

$$
A_{W}=U_{W} S_{W} V_{W}^{T}
$$

- Singular values of each sub-band are modified by the singular values of the DWT transformed visual watermark:

$$
s_{i}^{* k}=s_{i}^{k}+\alpha s_{w i}, \quad i=1, \ldots, n,
$$

where $s_{i}^{k}$ are singular values of $S^{k}, s_{w i}$ are singular values of $S_{w}$ and $\alpha$ is the scale factor.

- Finally 4 sets of modified DWT/DCT coefficients are obtained by the equation

$$
A^{* k}=U_{A}^{k} S_{A}^{* k} V_{A}^{k T},
$$

where $k=1,2,3,4$

and the watermarked image can be obtained using inverse 2DDWT / 2-DCT.

Watermark extraction process (Fig. 2) can be summarized in the following steps:

- The watermarked image is divided into 4 sub-images $I_{w w}, \ldots, I_{w 4}$.

- The 2D-DWT (only the first decomposition) or 2D-DCT is applied on each sub-image $I_{w 1}, \ldots, I_{w 4}$.

- The SVD is applied on each of the CA sub-bands in the case of 2D-DWT or on the first quarter of the zig-zag scanned coefficients in case of DCT.

$$
A=U S V^{T}
$$

- The 2D-DWT/2D-DCT is applied on the entire visual watermark $W$.

- The singular values are extracted from each quadrant $A_{k}$, where $k=1,2,3,4$

$$
s_{\text {wei }}^{k}=\left(s_{i}^{* k}-s_{i}^{k}\right) / \alpha, \quad i=1, \ldots, n,
$$




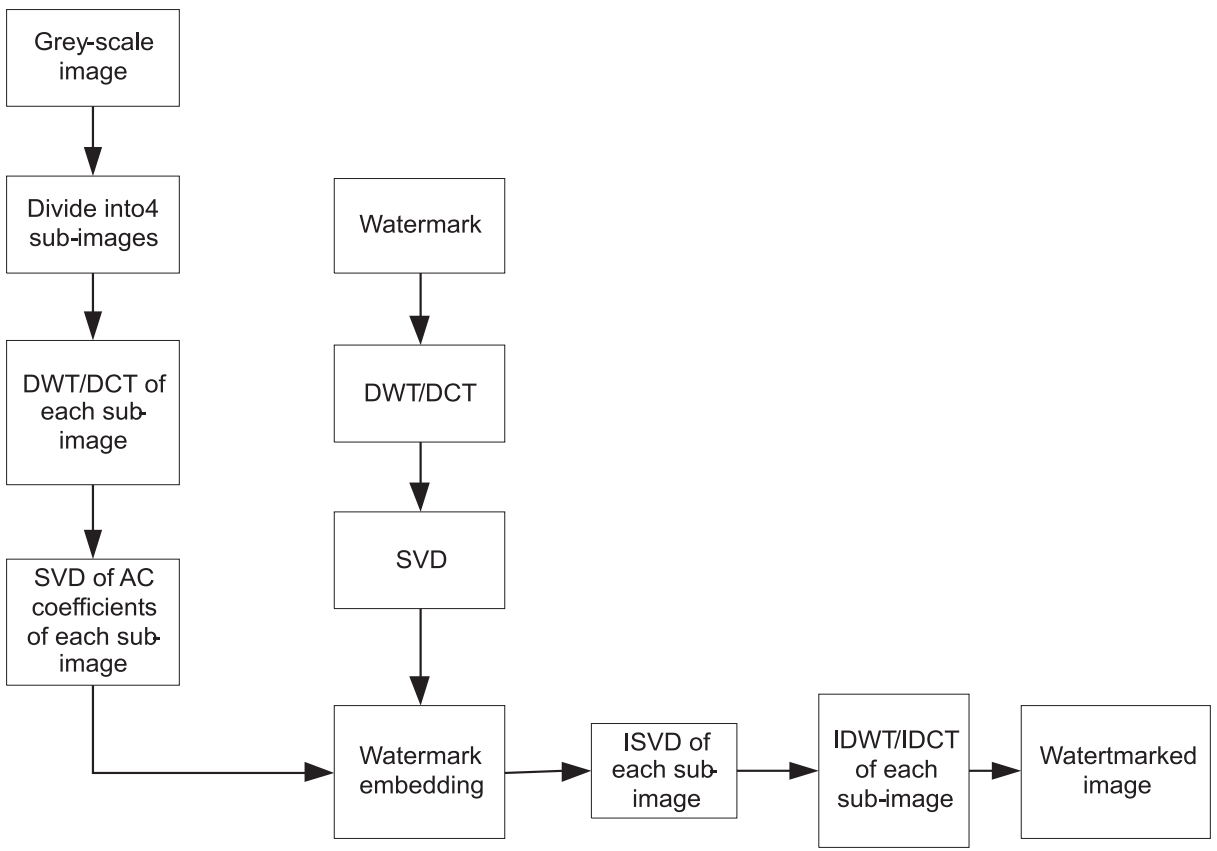

Fig. 1 Watermark embedding process

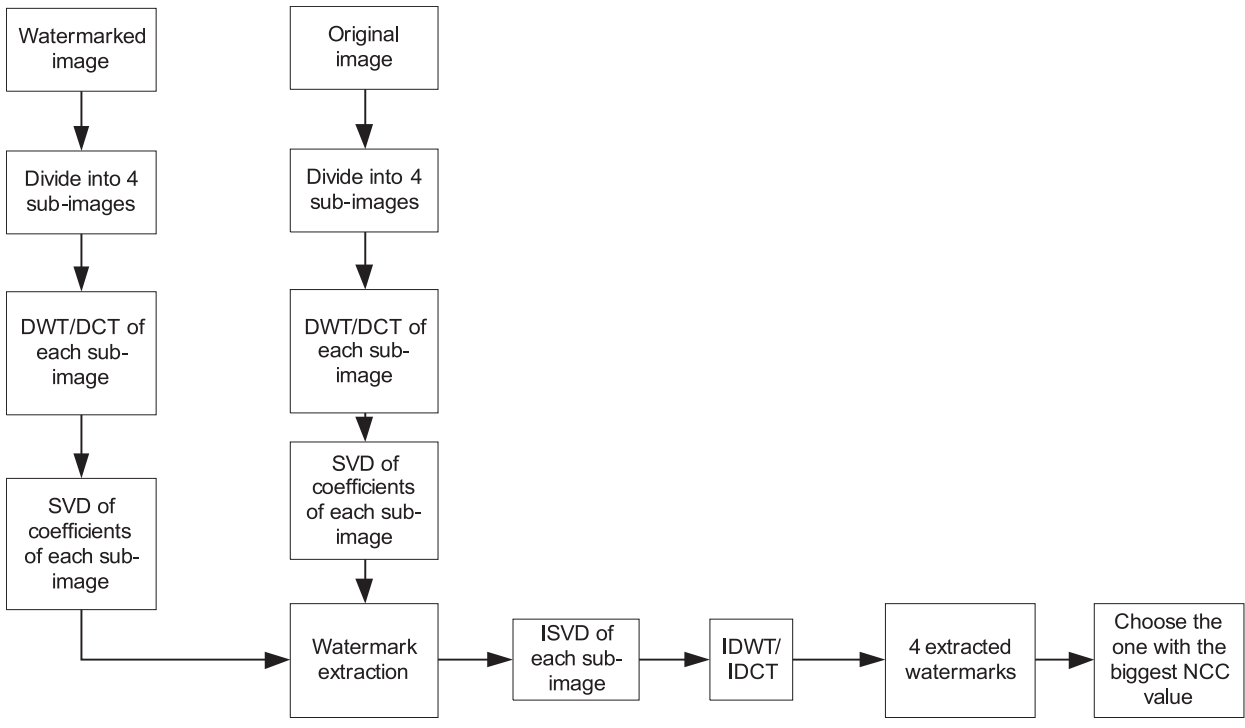

Fig. 2 Watermark extraction

where $s_{w e i}^{k}$ are singular values of extracted watermark $s_{w e}^{k}$ in $k$ quadrant.

- The inverse 2D-DWT or 2D-DCT is applied on each set to construct four extracted watermark images.

- The best extracted watermark is chosen according to the computed NCC.

\section{Evaluation of the proposed methods}

We use the Checkmark benchmark tool to evaluate the proposed methods. The Checkmark easily carries out attacks on watermarked image and returns the results of watermark extraction success.

There exist 4 groups of attacks in the Checkmark: removal attacks, geometric attacks, cryptographic attacks and protocol 


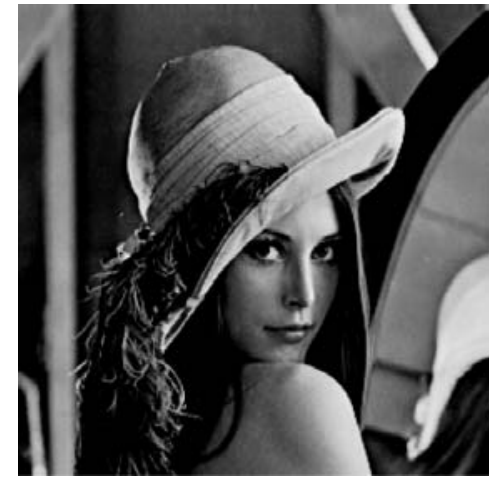

a)

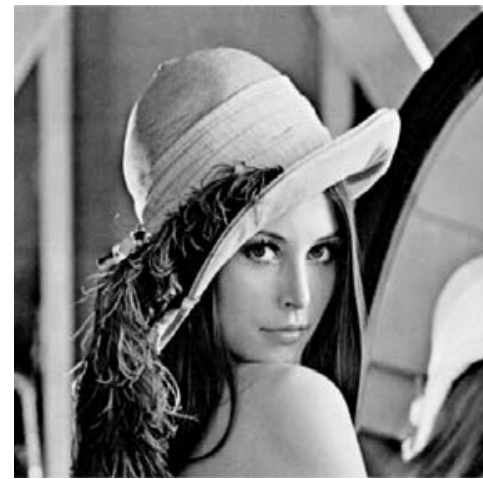

b)

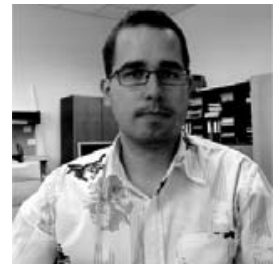

c)

Fig. 3 a) Lenna original, b) Lenna - watermarked image, c) Watermark

attacks. The image Lenna (Fig. 3a) as the original image I and the image of face (Fig. 3c) as the watermark image were used to test the proposed methods. The scale factor 0.16 was used as a tradeoff between watermark invisibility and robustness. The differences between the original and the watermarked image are shown in Figs. 3a) and 3b). The watermarked image (Fig. 3b) has notably different brightness than the original image (Fig. 3a).

We compared the novel method with a method that uses only DCT domain [10]. This method uses the luminance part of the image and DCT to embed the watermark in the image. Table 1 shows the results of watermark extraction after the selected attacks. 384 were carried out in total. However, Table 1 shows only the most common and most frequently used attacks.

\section{Conclusion}

The paper describes newly proposed image watermarking methods based on the wavelet transform and disrete cosine transform and singular value decomposition and. Our methods were compared with method described in [10]. The results introduced at the end of this paper show that our methods are highly robust to a lot of attacks.. The advantage of the proposed methods lies in high percentage of correctly extracted watermarks. The disadvantage of both methods is high computational complexity. We would like to improve these methods and add error correction codes to improve the reliability in the future.

\section{Acknowledgment}

This work was financially supported by the SIX Centre, OPVVI fund number CZ.1.05/2.1.00/03.0072 sponsored by the Ministry of Education, Youth and Sports of the Czech Republic and European Union.
Results of the test

Table 1

\begin{tabular}{|c|c|c|c|}
\hline Attacks & NCC - DCT & NCC - DWT & NCC- \\
\hline Cropping $10 \%$ & 0.9627 & 0.9178 & \\
\hline Cropping $20 \%$ & 0.8863 & 0.8728 & \\
\hline Cropping 50\% & 0.7245 & 0.7357 & \\
\hline Cropping $75 \%$ & 0.6301 & 0.6046 & \\
\hline Dithering & 0.7840 & 0.6966 & \\
\hline Gaussian blur $3 \times 3$ & 0.9872 & 0.9899 & 0.8264 \\
\hline Gaussian blur $5 \times 5$ & 0.9870 & 0.9897 & 0.8264 \\
\hline JPEG q $=10$ & 0.9974 & 0.9911 & $>0.7000$ \\
\hline JPEG q $=40$ & 0.9995 & 0.9982 & $>0.7000$ \\
\hline JPEG q $=90$ & 0.9997 & 0.9999 & 0.8667 \\
\hline Median filtering $(3 \times 3)$ & 0.9813 & 0.9878 & \\
\hline Median filtering $(4 \times 4)$ & 0.9930 & 0.9575 & \\
\hline Ratio change (0.8:1) & 0.9890 & 0.9899 & \\
\hline Ratio change (1:0.8) & 0.9847 & 0.9945 & \\
\hline Ratio change (1:1.2) & 0.9903 & 0.9973 & \\
\hline Rotation $15^{\circ}$ & 0.8174 & 0.7234 & \\
\hline Rotation $-1^{\circ}$ & 0.7232 & 0.7570 & \\
\hline Rotation $45^{\circ}$ & 0.9880 & 0.9600 & \\
\hline Scale (0.50x) & 0.8169 & 0.9102 & \\
\hline Scale $(0.75 x)$ & 0.9656 & 0.9806 & \\
\hline Scale $(0.90 x)$ & 0.9722 & 0.9794 & \\
\hline Scale (1.10x) & 0.9865 & 0.9906 & \\
\hline Scale (1.50x) & 0.9969 & 0.9968 & \\
\hline Scale (2.00x) & 0.9950 & 0.9958 & \\
\hline Sharpening & 0.8316 & 0.8700 & \\
\hline Wiener filter $(3 \times 3)$ & 0.9718 & 0.9773 & \\
\hline Jpeg 2000 (0.1 bit/pix) & 0.9148 & 0.9110 & \\
\hline Jpeg 2000 (3.5 bit/pix) & 0.9998 & 0.9997 & \\
\hline Jpeg 2000 (8.0 bit/pix) & 1.0000 & 0.9997 & \\
\hline
\end{tabular}




\section{References}

[1] COX, J., MILLER, M. L., BLOOM, J. A.: Digital Watermarking, Ed. London: Academic Press, 2002, ISBN 1-55860-714-5

[2] DARMSTAEDTER, J. DELAIGLE, J. QUISQUATER, M. B.: Low-cost Spatial Watermarking. Computer\&Graphics. vol. 33, No. 4, 1998, p. 417-424, ISSN 0097-8493

[3] CIKA, P.: New Watermarking Scheme for Colour Image. 12 ${ }^{\text {th }}$ Intern. Conference on Personal Wireless Communications (PWC 2007), Prague, , vol. 245, 2007

[4] HARGAS, L.: Application of Communication Systems in Biomedical Engineering, 2006, Communications - Scientific Letters of the University of Zilina, No. 1, p. 43-47, 2006, ISSN 1335-4205

[5] SVERDLOV, X., DEXTER, S., ESKICIOGLU, A. M.: Robust DCT/SVD Domain Image Watermarking for Copyright Protection: Embedding Data in all Frequencies, EUSIPCO, Antalya, 2005

[6] CIKA, P., ZUKAL, M.: Efficiency of BCH Codes in Digital Image Watermarking, $35^{\text {th }}$ Intern. Conference on Telecommunications and Signal Processing (TSP), Prague, 2012

[7] CIKA, P., KOVAR, P., KOHOUTEK, M.: Improved Digital Image Watermarking Method Based on DWT and SVD, $31^{\text {st }}$ International Conference on Telecommunications and Signal Processing (TSP 2008), Paradfurdo, 2008

[8] CIKA, P.: Watermarking Scheme Based on Discrete Wavelet Transform and Error - Correction Codes, $16^{\text {th }}$ Intern. Workshop on Systems, Signals and Image Processing (IWSSIP 2009), Chalkida, pp. 250-253, 2009

[9] VOLOSHYNOVSKIY, P. S., MADUENO, M., MARCHAND-MAILLET, S., PUN, T.: Second Generation Benchmarking and Application Oriented Evaluation, Information Hiding Workshop, Pittsburgh, April 2001.

[10] MENG Z, YU P, YU G. Copyright Protection for Digital Image Based on Joint DWT-DCT Transformation, Wavelet Analysis and Pattern Recognition (ICWAPR), 2012 pp.11-14, 15-17 July 2012 doi: 10.1109/ICWAPR.2012.6294746. 